\title{
Appearance of estrogen positive bilateral BREAST CARCINOMA WITH HER 2 GENE AMPLIFICATION IN A PATIENT WITH APLASTIC ANEMIA
}

\author{
Milica Čolović ${ }^{1}$, Milena Todorović ${ }^{1,2}$, Natasa Čolović ${ }^{1,2}$, Tatjana Terzic $^{1}$, Katarina Karadzic ${ }^{3}$, \\ Vladimir Jurišić ${ }^{4}$
}

${ }^{1}$ Faculty of Medicine, University of Belgrade, Belgrade, Serbia

${ }^{2}$ Clinic for Hematology, Clinical Center for Serbia, Belgrade, Serbia

${ }^{3}$ Institute of Oncology and Radiology of Serbia, Belgrade, Serbia

${ }^{4}$ University of Kragujevac, Faculty of Medical Sciences, Kragujevac, Serbia

\begin{abstract}
Immunosuppressive therapy is one of the standard therapy protocols for aplastic anemia (AA). However, immunosuppressive therapy and androgenic steroids can promote development of solid tumors such as squamous carcinoma, head and neck tumors, adenocarcinoma of the stomach, hepatocarcinoma and breast carcino$\mathrm{ma}$ in long surviving patients with aplastic anemia. We present here a rare case of a 56-year-old woman in whom bilateral adenocarcinoma of the breast developed 11 years after the start of immunosuppressive and androgenic steroid therapy for aplastic anemia. Histological examination showed invasive ductal carcinoma with intense nuclear staining for estrogen receptors. HER2 immunohistochemistry was positive for $80 \%$ of stained cells, and chromogenic in situ hybridization showed a high level of HER2 gene amplification. This case indicated that a new therapy option is needed for estimation and evaluation to avoid the consequence of cancer occurrence.
\end{abstract}

Key words: aplastic anemia, immunosuppressive therapy, breast cancer, histology, immunohistochemistry, estrogen receptors, HER2, survival, cancer, chromogenic in situ hybridization (CISH).

\section{Introduction}

It is well known that long-term survivors of acquired aplastic anemia (AA) may develop hematologic or non-hematologic malignant diseases [1-3]. Immunosuppressive therapy with or without antithymocyte globulin and cyclosporine $A$ is the standard treatment for aplastic anemia $[1,3]$.

In addition, the risk of acute leukemia is very high especially in those patients who received multiple courses of immunosuppressive chemotherapy during treatment of aplastic anemia [3, 4]. The risk for solid malignant tumors is lower but there have been ob- served squamous carcinoma of the neck and head, adenocarcinoma of the stomach, hepatocarcinoma and breast tumors [5]. The development of breast cancer has been reported more frequently after treatment of Hodgkin's lymphoma [6]. Here we report a case of bilateral breast carcinoma in a 56-year-old woman which developed 11 years after treatment of aplastic anemia with immunosuppressive therapy and androgens.

\section{Case report}

A 46-year-old woman presented in December 1996 with profound hemorrhagic syndrome mani- 
fested with diffuse hematomas, petechial bleeding and metrorrhagia. Her past medical history was unremarkable. She had no medications, exposures to chemicals or viral symptoms. Laboratory data were as follows: $\mathrm{Hb} 52 \mathrm{~g} / \mathrm{l}$, platelets $6 \times 10^{9} / 1$, white blood cells (WBC) $0.7 \times 10^{9} / 1$ (lymphocytes $85 \%$, monocytes $15 \%$ ). Biochemical analyses were normal. Bone marrow aspirate showed severe hypocellularity with rare normal cells and histopathology examination of bone marrow confirmed a diagnosis of aplastic anemia (Fig. 1). The culture of hematopoietic progenitor cells from a bone marrow sample revealed very low growth of CFU-GM and GM clusters. CFU-E and BFU-E were not found and there was also absence of growth of CFU-Mk, which was in correlation with aplastic anemia. Chest radiography and abdominal ultrasound findings were normal. Flow cytometry for paroxysmal nocturnal hemoglobinuria was negative. At that time the patient was treated with packed red blood cells and vitamin $\mathrm{B}_{12}$. After excluding paroxysmal nocturnal hemoglobinuria and confirming the diagnosis of aplastic anemia she was treated with antithymocyte globulin (ATG) in continuous infusion for 5 days (she received 50 flacons with $25 \mathrm{mg}$ of protein per vial) and corticosteroids $100 \mathrm{mg} / \mathrm{per}$ day. The treatment continued with cyclosporin A. She developed steroid diabetes which resolved after corticosteroid discontinuation. After 6 months she recovered completely. Bone marrow aspirate and histology showed normal but slightly hypocellular hematopoiesis. After one year cyclosporin was discontinued and she was well with the following findings: $\mathrm{Hb} 117 \mathrm{~g} / \mathrm{l}$, platelets $56 \times 10^{\circ} / \mathrm{l}$, WBC $2.5 \times 10^{9} / 1$ (lymphocytes $29 \%$, nibicytes $16 \%$ neutrophils $53 \%$ ). She was without therapy and in very good condition until the end of 2009 when she noticed a palpable tumor in the right breast. In February 2010 a radical right mastectomy was performed and pathohistology revealed invasive ductal carcinoma (IDC) (in diameter $26 \times 22 \times 25 \mathrm{~mm}$ ) and in March 2010 a left quadrantectomy of the breast was done. Again histology showed carcinoma. After, bilateral modified radical mastectomy was performed. Serial sectioning revealed a firm yellow tan circumscribed mass located in the left breast measuring $11 \times 10 \times$ $6 \mathrm{~mm}$ and a mass located in the right breast measuring $26 \times 22 \times 25 \mathrm{~mm}$. The largest node measured 16 $\times 10 \times 6 \mathrm{~mm}$. The diagnosis was obtained on review of the hematoxylin and eosin-stained slides. Both tumors showed similar histological features (Fig. 2) and the diagnosis was invasive ductal carcinoma (histological grade 2, nuclear grade II) with nodal metastases ( 1 of 13 left axillary lymph nodes and 17 of 18 right axillary lymph nodes). Left breast cancer specimens underwent HER2/neu (human epidermal growth factor receptor 2) testing by a validated immunohistochemistry (IHC) assay (i.e., HercepTest, Dako,
Glostrup, Denmark). HER2 expression was 3+ (uniform intense membrane staining of more than $80 \%$ of tumor cells). Estrogen receptor (ER) and progesterone receptor (PR) were negative. Right breast cancer specimens showed HER2 expression $(2+)$, positive estrogen receptor (ER) (score 7), but negative progesterone receptor (PR). At that time $\mathrm{CBC}$ was normal and histology of bone marrow detected normal hematopoiesis with cellularity of $40 \%$ and without presence of non-hematopoietic cells. In vitro cell cultures showed that the number of CFU-GM colonies was slightly lowered and after stimulation the number CFU-E and BFU-E was normal. Hematology analysis was normal including $\mathrm{Hb} 122 \mathrm{~g} / \mathrm{l}$, WBC $4.0 \times 10^{9} / \mathrm{l}$, and platelets $171 \times 10^{9} / \mathrm{l}$. The oncology team suggested chemotherapy with doxorubicin in a dose of $50 \mathrm{mg} / \mathrm{m}^{2}$ and cyclophosphamide in a dose of $500 \mathrm{mg} / \mathrm{m}^{2}$. She was treated with 4 courses of chemotherapy after which she was neutropenic for a while and neutropenia resolved with G-CSF, but in spite of all treatment one year after she developed multiple metastases in bones and disease progression.

\section{Discussion}

Acquired aplastic anemia is a rare hematopoietic disease in which bone marrow does not produce an adequate number of blood cells, leading to pancytopenia. It can be treated with bone marrow transplantation or immunosuppressive therapy and steroid androgens $[1,2]$. Survival is better after bone marrow transplantation but immunosuppressive therapy also

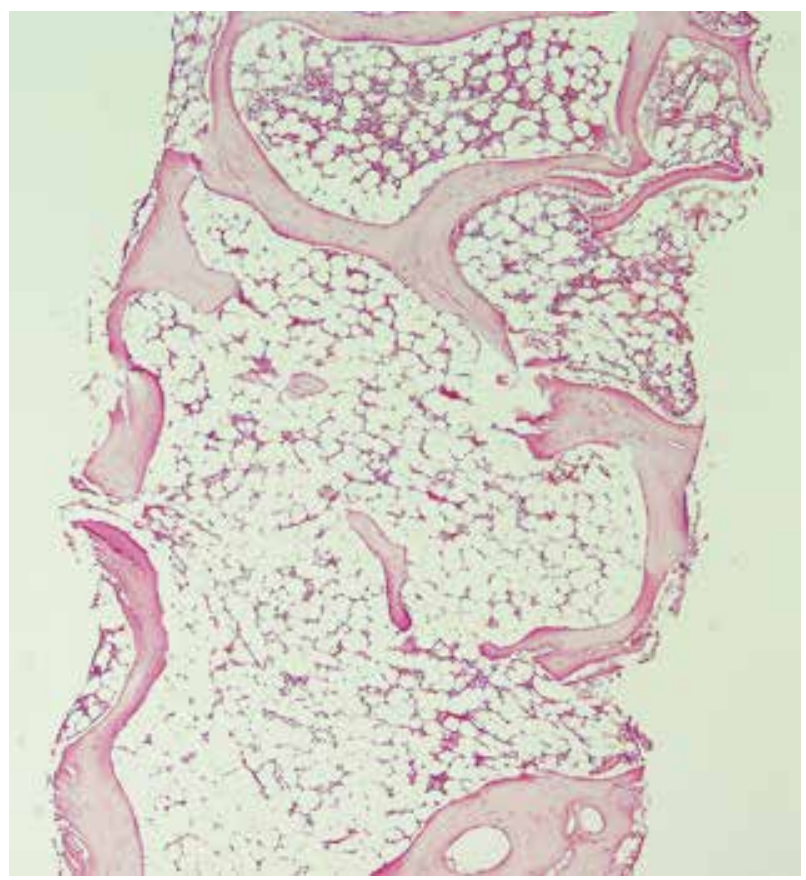

Fig. 1. Bone marrow trephine biopsy section, aplastic anemia, showed marked hypocellularity (hematoxylin and eo$\sin , 40 \times)$ 

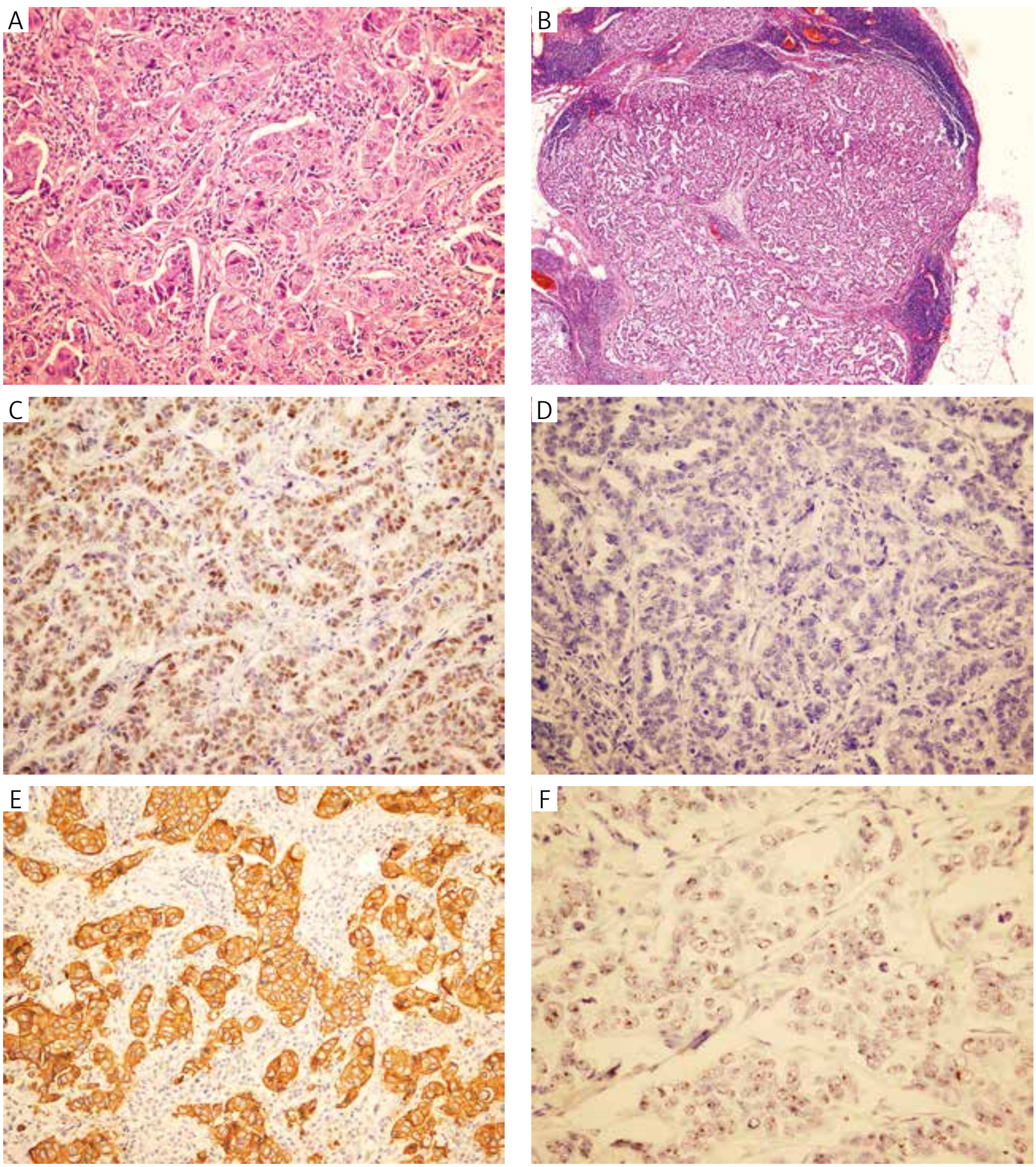

Fig. 2. Breast cancer. A) Histologic examination of the breast tumor showed invasive ductal carcinoma infiltrating predominantly in sheets with occasional glandular formations (hematoxylin and eosin, 200×). B) Nodal metastasis of breast carcinoma (hematoxylin and eosin, $40 \times$ ). C) Right breast cancer specimens showed intense nuclear staining for estrogen receptor in the vast majority of cells - Allred score category $7(200 \times)$. D) Tumor cells were negative for progesterone receptor $(200 \times)$. E) Immunohistochemistry for HER2 in left breast cancer specimens (uniform intense membrane staining of more than $80 \%$ of tumor cells) $(200 \times)$. F) Chromogenic in situ hybridization (CISH) showing high level of HER2 gene amplification $(400 \times)$

leads to long-term survival in almost $60 \%$ of patients. Since the number of long-term survivors of aplastic anemia is increased, the complications of therapy, especially malignant hematologic and non-hematologic diseases, appeared as a major problem of concern
[4, 6-8]. Najean reported in 1990 five cases of acute leukemia among 156 patients with aplastic anemia who were treated only with androgens, raising the question whether aplastic anemia is a preleukemic syndrome [9]. The hematologic and non-hematologic 
post-treatment complications have been studied in a large cohort of 1608 cases reported to the European Bone Marrow Transplantation-Severe Aplastic Anaemia (EBMT-SAA) Working Party over a 20-year period. They found that survivors of aplastic anemia are at high risk for hematologic malignancies such as myelodysplastic syndrome and acute leukemias. The majority of such cases developed hematologic malignancy after immunosuppressive therapy while the incidence of solid tumors is similar after immunosuppression and after bone marrow transplantation [7]. In this large group of patients there are only two patients with breast carcinoma who were treated with immunosuppressive therapy.

However, another study showed that 15-year cumulative incidence for any cancer in long-term survivors of aplastic anemia treated with allogeneic bone marrow transplantation was $10.9 \%$ [10, 11]. There are occasional cases of aplastic anemia and breast cancer treated with androgenic steroids [3] explaining the occurrence due to existence of sex hormone receptors.

In this paper we documented a very rare case with bilateral breast carcinoma positive for sex steroid receptors and HER2 that developed after 11 years of immunosuppressive therapy.

Authors declare no conflict of interests. This paper is supported by part of scientific project number 175056, financed by the Ministry of Sciences and Education of the Republic of Serbia.

\section{References}

1. Sakamoto T, Obara N, Kurita N, et al. Effectiveness and safety of rabbit anti-thymocyte globulin in Japanese patients with aplastic anemia. Int J Hematol 2013; 98: 319-322.

2. Meidlinger P, Knöbl P, Jäger U, et al. Granulocyte colony-stimulating factor-supported combined immunosuppressive therapy (antilymphocyte globulin, cyclosporine, and methylprednisolone) in patients with aplastic anemia: tolerability, efficacy, and changes in the progenitor cell compartment. Ann Hematol 1999; 78: 299-304.

3. Fujino Y, Ku Y, Suzuki Y, et al. Ampullary carcinoma developing after androgenic steroid therapy for aplastic anemia: report of a case. Surgery 2001; 129: 501-503.

4. Socié G, Henry-Amar M, Bacigalupo A, et al. Malignant tumors after treatment of aplastic anemia. N Engl J Med 1993; 329: 1152-1157.

5. Enjeti AK, Seldon M, Braye S. Bilateral breast lumps in a patient after sex mismatched allogeneic transplantation for aplastic anaemia. Clin Pathol 2005; 58: 670-671.

6. Kaldor JM, Day NE, Band P, et al. Second malignancies following testicular cancer, ovarian cancer and Hodgkin's disease: an international collaborative study among cancer registries. Int J Cancer 1987; 39: 571-585.

7. Socié G, Henry-Amar M, Cosset JM, et al. Increased incidence of solid malignant tumors after bone marrow transplantation for severe aplastic anemia. Blood 1991; 78: 277-279.

8. Witherspoon RP, Storb R, Pepe M, et al. Cumulative incidence of secondary malignant tumors in aplastic anemia patients giv- en marrow grafts after conditioning with chemotherapy alone. Blood 1992; 79: 289-291.

9. Najean Y, Haguenauer O. Long term (5 to 20 years) evolution of nongrafted aplastic anemias. Blood 1990; 76: 2222-2228.

10. Ades L, Mary JY, Robin M, et al. Long-term outcome after bone marrow transplantation for severe aplastic anemia. Blood 2004; 103: 2490-2497.

11. Shea B, Reddy V, Abbitt P, et al. Granulocyte sarcoma (chloro$\mathrm{ma}$ ) of the breast: a diagnostic dilemma and review of literature. Breast J 2004; 10: 48-53.

\section{Address for correspondence}

Prof. Dr Vladimir Jurisic

University of Kragujevac

Faculty of Medical Sciences PO BOX 124

34-000 Kragujevac, Serbia tel. +3813430680

e-mail:vdvd@mailcity.com 